\title{
Meios para dissolução de comprimidos de nimesulida: ação dos tensoativos
}

\author{
Regina Lengruber da Silva, Nadia Maria Volpato* \\ Departamento de Medicamentos, Faculdade de Farmácia, Universidade Federal do Rio de Janeiro
}

*Correspondência:

N. M. Volpato

LabCQ - Depto. de Medicamentos -

Faculdade de Farmácia da UFRJ

Centro de Ciências da Saúde, Bloco

Bss salas 24 a 34 - Cidade

Universitária

21941-590 Rio de Janeiro RJ - Brasil

E-mail: volpato@pharma.ufrj.br
A nimesulida é um antiinflamatório não-esteróide com baixa solubilidade em água e caráter fracamente ácido. $O$ desenvolvimento dos ensaios de dissolução para fármacos de baixa hidrossolubilidade pode ser problemático e tensoativos são freqüentemente requeridos para aumentar a solubilização dos mesmos. No presente trabalho avaliou-se o efeito dos tensoativos lauril sulfato de sódio e polissorbato 80 , na promoção da solubilidade da nimesulida em meio aquoso, determinando-se também o $\mathrm{pH}$, a tensão superficial e a concentração micelar crítica dos meios testados. A nimesulida apresentou baixa solubilidade nos meios sem tensoativos ( $<60 \mu \mathrm{g} / \mathrm{mL}$ ), na faixa de $\mathrm{pH}$ de 1,0 a 7,4 e as maiores solubilidades (>300 $\mu \mathrm{g} / \mathrm{mL}$ ) foram observadas em tampão fosfato $\mathrm{pH}$ 7,4 com polissorbato 80. A concentração de saturação do fármaco aumentou proporcionalmente com a concentração dos tensoativos, os quais atuam pelo mecanismo de solubilização micelar. Obtiveram-se perfis de dissolução de três formulações de comprimidos de nimesulida, em meios com diversas concentrações de tensoativo, empregando-se o aparelho de dissolução com agitador de pá. Os resultados mostraram que a escolha do meio de dissolução para nimesulida deve ser criteriosa, de modo a evitar condições de baixo poder discriminante das formulações.
Unitermos:

- Nimesulida

- Dissolução

- Tensoativo

- Solubilidade

- Tensão superficial

\section{INTRODUÇÃO}

A nimesulida (Figura 1) é um antiinflamatório nãoesteróide com propriedades analgésicas e antipiréticas (Davis, Brogden, 1994), que possui caráter fracamente ácido (pKa $\cong 6,5$; Singh, Sharda, Mahajan, 1999) e baixa solubilidade em água ( $\cong 10 \mu \mathrm{g} / \mathrm{mL}$; Piel et al., 1997). A nimesulida pertence à categoria II da Classificação Biofarmacêutica (baixa solubilidade, alta permeabilidade) (Amidon et al., 1995; Bernareggi, 1998) e, para tais moléculas, a dissolução in vivo pode vir a ser o passo limitante da absorção oral do fármaco. Nestes casos, o teste de dissolução in vitro assume papel fundamental no controle de qualidade de formulações sólidas, de modo a assegurar a biodisponibilidade e promover a homogeneidade inter-lotes. Até o presente momento, constatase ausência de monografia para produto acabado de nimesulida nos principais compêndios oficiais.

A solubilidade intrínseca de um fármaco desempenha importante papel na sua dissolução a partir de uma forma farmacêutica sólida. Logo, torna-se fundamental conhecer a solubilidade do mesmo em diferentes 


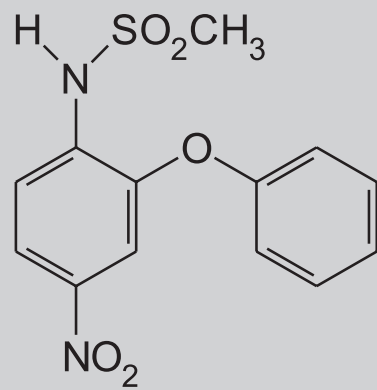

FIGURA 1- Estrutura química da nimesulida (NMS).

solventes, de modo a selecionar meios de dissolução adequados para as formulações que o contenham.

Para fármacos de baixa hidrossolubilidade e de caráter fracamente ácido, como é o caso da nimesulida, algumas dificuldades apresentam-se na seleção de meios para dissolução, de modo que estes propiciem, na medida do possível, a "condição sink", sem, necessariamente, envolver um grande volume de meio ou um mecanismo de substituição constante do mesmo (Cárcamo, 1981; Abdou, 1995; El-Massik et al., 1996; CDER/FDA, 1997).

Para aumentar a solubilidade de fármacos sensíveis ao $\mathrm{pH}$, pode-se alterar o $\mathrm{pH}$ do meio ou introduzir modificadores como, por exemplo, tensoativos ou solventes orgânicos. Se a mudança de $\mathrm{pH}$ está inserida na faixa fisiológica, a solubilidade e os dados de dissolução obtidos podem ser empregados no estabelecimento de uma correlação in vivo-in vitro (CDER/FDA, 1997). O emprego de $\mathrm{pH}$ fora dos limites fisiológicos torna esta correlação inadequada, sendo sua adoção desaconselhada, do mesmo modo que é o uso de solventes orgânicos (Löbenberg, Amidon, 2000).

O uso de tensoativos em meios de dissolução é uma das principais formas para aumentar a solubilidade de fármacos insolúveis ou ligeiramente solúveis em água (Shah et al., 1995; Amidon et al., 1995; Storey, 1996), sendo apropriado devido à possibilidade de simular o ambiente in vivo do lúmen intestinal (Galia et al., 1998). Os tensoativos endógenos, lecitina e ácidos e sais biliares, têm mostrado aumentar o grau de dissolução de fármacos de baixa hidrossolubilidade, pelo fato de os mesmos serem solubilizados no organismo antes de serem absorvidos.

Os mecanismos mais prováveis de atuação dos tensoativos são a molhabilidade e a solubilização micelar (Schott, Kwan, Feldman, 1982). O processo de molhabilidade consiste em deslocar uma microfase de ar da superfície do sólido e substituí-la por uma microfase líquida, diminuindo o ângulo de contato sólido-líquido e as tensões superficial e interfacial do meio aquoso (Schott, Kwan, Feldman, 1982; Martin, 1993a; Zografi, 1995), aumentando, por conseguinte, o processo de penetração do meio dissolvente na matriz sólida (Abdou, 1995). No mecanismo de solubilização micelar, o tensoativo adicionado encontra-se em concentração superior à concentração micelar crítica (CMC). Logo, há formação de micelas no seio do líquido (pseudofase) e o fármaco, pouco hidrossolúvel, pode estar dissolvido no interior das mesmas, originando um meio aquoso aparentemente homogêneo (Schott, Kwan \& Feldman, 1982; Martin, 1993b; Schott, 1995; Holland et al., 1997). Acima da CMC, a tensão superficial permanece essencialmente constante, indicando que a interface sólido-líquido está saturada e a formação de micelas teve lugar no meio líquido (Martin, 1993a).

Devido ao maior custo dos tensoativos naturais ou endógenos, não é prático utilizá-los nos estudos de dissolução, podendo os mesmos ser substituídos por tensoativos sintéticos, como o lauril sulfato de sódio e o polissorbato 80 (Shah et al., 1995), os quais são os tensoativos mais freqüentemente utilizados pela United States Pharmacopeia (2000), nas monografias atualmente constantes. Baixos níveis dos mesmos $(0,5-5,0 \%$ p/v) são indicados para serem incluídos no meio de dissolução, de maneira a fornecer melhor relação com as condições in vivo, principalmente no estado de jejum (Abdou, 1995).

A proposta do presente trabalho foi avaliar o efeito dos tensoativos lauril sulfato de sódio (LSS) e polissorbato 80 (Poli 80) na solubilidade da nimesulida, em diferentes meios propostos para dissolução (diversos $\mathrm{pH}$, tensoativos em diferentes concentrações), bem como averiguar sua influência no perfil de dissolução de comprimidos de nimesulida de diferentes fabricantes.

\section{MATERIAL E MÉTODOS}

\section{Solubilidade da nimesulida em diferentes meios}

Os meios testados na avaliação da solubilidade da nimesulida (gentilmente cedida pela Schering-Plough,

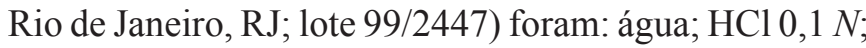
tampão fosfato $\mathrm{pH}$ 5,8 (United States Pharmacopeia, 2000); tampão fosfato $\mathrm{pH} \mathrm{7,4} \mathrm{(United} \mathrm{States}$ Pharmacopeia, 2000); soluções aquosas contendo lauril sulfato de sódio $(0,5 ; 1,0 ; 1,5 ; 2,0$ e $2,5 \% \mathrm{p} / \mathrm{v})$; soluções aquosas contendo polissorbato $80(0,5 ; 1,0 ; 1,5 ; 2,0 \mathrm{e}$ $2,5 \% \mathrm{p} / \mathrm{v}) ; \mathrm{HCl} 0,1 \mathrm{~N}$ contendo LSS (1,0 e 2,0\% p/v); HCl $0,1 N$ contendo Poli $80(1,5$ e $2,5 \%$ p/v); tampão fosfato pH 5,8 contendo Poli $80(1,5 ; 2,0$ e $2,5 \%$ p/v); tampão fosfato $\mathrm{pH} 7,4$ contendo Poli $80(1,5 ; 2,0$ e $2,5 \% \mathrm{p} / \mathrm{v})$; tam- 
pão fosfato $\mathrm{pH} 7,4$ contendo LSS (1,0 e 2,0\% p/v). Este último tampão foi preparado com os sais monoidrogeno e diidrogeno fosfato de sódio e não de potássio, normalmente utilizado, em função da incompatibilidade física entre os íons potássio e o LSS (Wade, Weller, 1994).

A solubilidade da nimesulida nos meios foi determinada pelo preparo de suspensão do fármaco nos mesmos, em tubo de ensaio, a qual foi mantida sob agitação magnética por 24 horas, a $37^{\circ} \mathrm{C} \pm 0,5^{\circ} \mathrm{C}$ e quantificando a substância ativa no sobrenadante por espectrofotometria de absorção no UV.

\section{Análise quantitativa da nimesulida por espectrofotometria de absorção no UV}

Em função da diferente composição dos meios empregados, o comportamento espectrométrico do fármaco nos mesmos foi avaliado através de seus espectros de absorção na região do UV (Shimadzu UV, modelo 1601). Selecionou-se o comprimento de onda de máxima absorção $\left(\lambda_{\max }\right)$ para cada caso e a quantificação foi realizada empregando-se curva padrão de absorvância versus concentração de nimesulida ( 5 a $40 \mu \mathrm{g} / \mathrm{mL}$ no meio dissolvente correspondente).

\section{Determinação do pH dos meios}

$\mathrm{O}$ pH dos meios foi determinado através de potenciômetro (Digimed, modelo DM21), previamente calibrado com soluções tampão pH 7,0 e pH 4,0 (QM Reagentes), à temperatura ambiente, com o intuito de verificar se os mesmos não sofriam alteração após adição dos tensoativos e encontravam-se dentro da faixa fisiologicamente relevante, como é aconselhado pelo CDER/ FDA (1997).

\section{Determinação da tensão superficial e da concentração micelar crítica (CMC) dos meios}

A tensão superficial dos meios foi determinada através de tensiômetro (Kruss, modelo K-10), à temperatura ambiente, de modo a estabelecer analogia entre a condição in vitro e a fisiológica (tensão superficial do suco gástrico entre 35 e 50 mN/m) (Finholt, Solvang, 1968).

A concentração micelar crítica (CMC) foi determinada nas soluções aquosas de LSS, Poli 80, tampão pH 7,4 contendo LSS e tampão pH 7,4 contendo Poli 80, com o objetivo de confirmar se as concentrações de tensoativos utilizadas encontravam-se acima da CMC. A CMC foi obtida, graficamente, a partir da tensão superficial de soluções diluídas do tensoativo em função de sua concentra- ção, determinando-se o ponto de inflexão da curva (Crison, Weiner, Amidon, 1997).

\section{Avaliação preliminar do perfil de dissolução de comprimidos de nimesulida}

A dissolução de comprimidos contendo $100 \mathrm{mg}$ de nimesulida foi avaliada a partir de três diferentes formulações (Produtos A, B e C), disponíveis no mercado nacional, empregando-se $1000 \mathrm{~mL}$ de determinados meios que forneceram menor (LSS - 0,5\%; Poli 80 - 1,5\%) e maior (LSS - 2,0\%; tampão pH 7,4 contendo Poli 80 - 1,5\%) concentração de saturação da nimesulida, conforme apresentado em Resultados e Discussão.

O ensaio foi realizado em aparelho de dissolução de cubas (Erweka, modelo DT6R), utilizando o agitador do tipo pá (aparato 2), na velocidade de $75 \mathrm{rpm}$. Os meios não foram desgaseificados devido à considerável formação de espuma, decorrente da presença dos tensoativos nas concentrações em estudo. A duração do ensaio foi de, no máximo, 3 horas, retirando-se alíquotas a intervalos de 15 minutos, 30 minutos ou 1 hora, para quantificação da nimesulida dissolvida, procedendo-se à reposição do meio imediatamente após a coleta. O doseamento foi realizado por espectrofotometria de absorção no UV, conforme descrito anteriormente, empregando-se sempre o respectivo meio de dissolução como solvente, comprimento de onda máximo correspondente, apresentado em Resultados e Discussão e curva padrão.

Os três produtos apresentaram-se em conformidade com as especificações gerais dos compêndios oficiais quanto ao teor (entre 90 e 110\%), uniformidade de peso, de dose, dureza e friabilidade (dados não apresentados), sendo que a desintegração para todos foi inferior a 2 minutos (em água a $37^{\circ} \mathrm{C} \pm 1{ }^{\circ} \mathrm{C}$ ).

\section{RESULTADOS E DISCUSSÃO}

\section{Análise quantitativa da nimesulida por espectrofotometria de absorção no UV}

Os comprimentos de onda de máxima absorção obtidos para a nimesulida nos meios para dissolução foram distintos e são apresentados na Tabela I.

Analisando estes resultados pode-se constatar que o solvente exerce forte influência nas características absortivas da molécula do fármaco. Esta influência não depende somente do $\mathrm{pH}$ resultante do meio (ver também Tabela III), uma vez que para iguais valores de $\mathrm{pH}$ obtiveram-se espectros diversos e, por outro lado, para valores muito diferentes de $\mathrm{pH}$ obtiveram-se espectros semelhantes, mas 
TABELA I - Comprimentos de onda de máxima absorção obtidos para a nimesulida nos meios para dissolução

\begin{tabular}{lc}
\hline Meios & Comprimento de onda $(\mathrm{nm})$ \\
\hline $\mathrm{H}_{2} \mathrm{O} ; \mathrm{HCl} \mathrm{0,1} \mathrm{N}$; Tampão & 299 \\
pH 5,8; todos os meios & \\
contendo LSS & \\
Todos os meios contendo & 335 \\
Poli 80, exceto Tampão & \\
pH 7,4 contendo Poli 80 & \\
Tampão pH 7,4 puro e & 398 \\
contendo Poli 80 &
\end{tabular}

depende, também, da própria composição qualitativa do meio.

\section{Solubilidade da nimesulida em diferentes meios}

Os resultados obtidos para a solubilidade da nimesulida nos meios avaliados são apresentados na Tabela II e ilustrados, parcialmente, na Figura 2.

Pelos dados apresentados, observa-se que a nimesulida apresentou baixa solubilidade nos meios sem tensoativo (da ordem de $10 \mathrm{mg} / \mathrm{mL}$ em água, $\mathrm{HCl} 0,1 \mathrm{Ne}$ tampão pH 5,8 e de $60 \mathrm{mg} / \mathrm{mL}$ em tampão $\mathrm{pH} 7,4)$ e que a maior solubilidade (em torno de $500 \mathrm{mg} / \mathrm{mL}$ ) foi

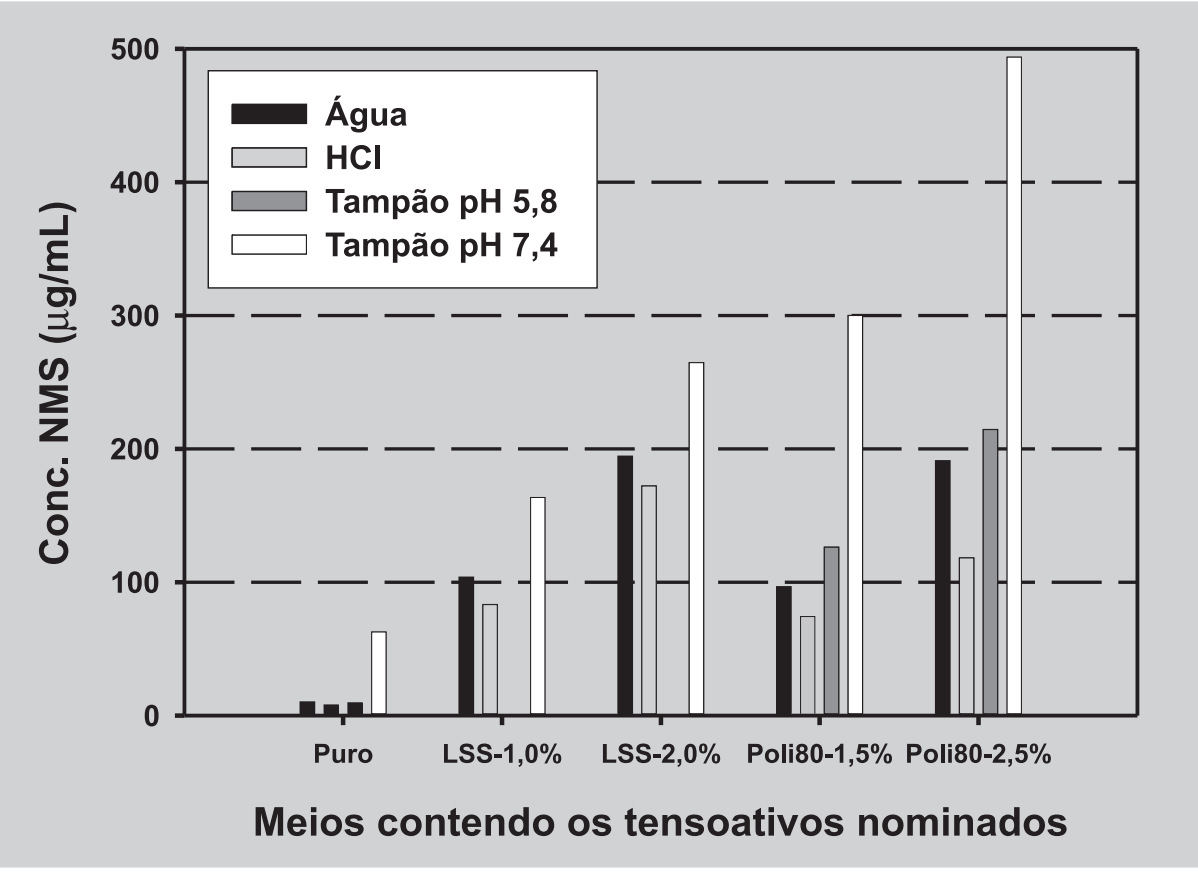

FIGURA 2 - Solubilidade da nimesulida (NMs) em diferentes meios, a $37{ }^{\circ} \mathrm{C} \pm 0,5{ }^{\circ} \mathrm{C}$.

TABELA II - Solubilidade da nimesulida (NMS) nos meios avaliados

\begin{tabular}{|c|c|c|c|c|c|}
\hline \multirow{2}{*}{\multicolumn{2}{|c|}{ Tensoativo Concentração (g\%) }} & \multicolumn{4}{|c|}{ Concentração NMS $(\mu \mathrm{g} / \mathrm{mL})$ média $(\mathrm{n}=2)$} \\
\hline & & Água & HCl $0,1 N$ & Tampão pH 5,8 & Tampão pH 7,4 \\
\hline \multirow{6}{*}{ LSS } & 0,0 & 10,1 & 7,9 & 9,5 & 62,6 \\
\hline & 0,5 & 56,7 & - & - & - \\
\hline & 1,0 & 103,6 & 83,2 & - & 163,4 \\
\hline & 1,5 & 155,4 & - & - & - \\
\hline & 2,0 & 194,4 & 172,0 & - & 264,6 \\
\hline & 2,5 & 287,4 & - & - & - \\
\hline \multirow{5}{*}{ Poli 80} & 0,5 & 28,2 & - & - & - \\
\hline & 1,0 & 62,5 & - & - & - \\
\hline & 1,5 & 96,6 & 74,2 & 126,2 & 300,0 \\
\hline & 2,0 & 155,0 & - & 166,5 & 395,8 \\
\hline & 2,5 & 191,0 & 118,2 & 214,3 & 493,6 \\
\hline
\end{tabular}




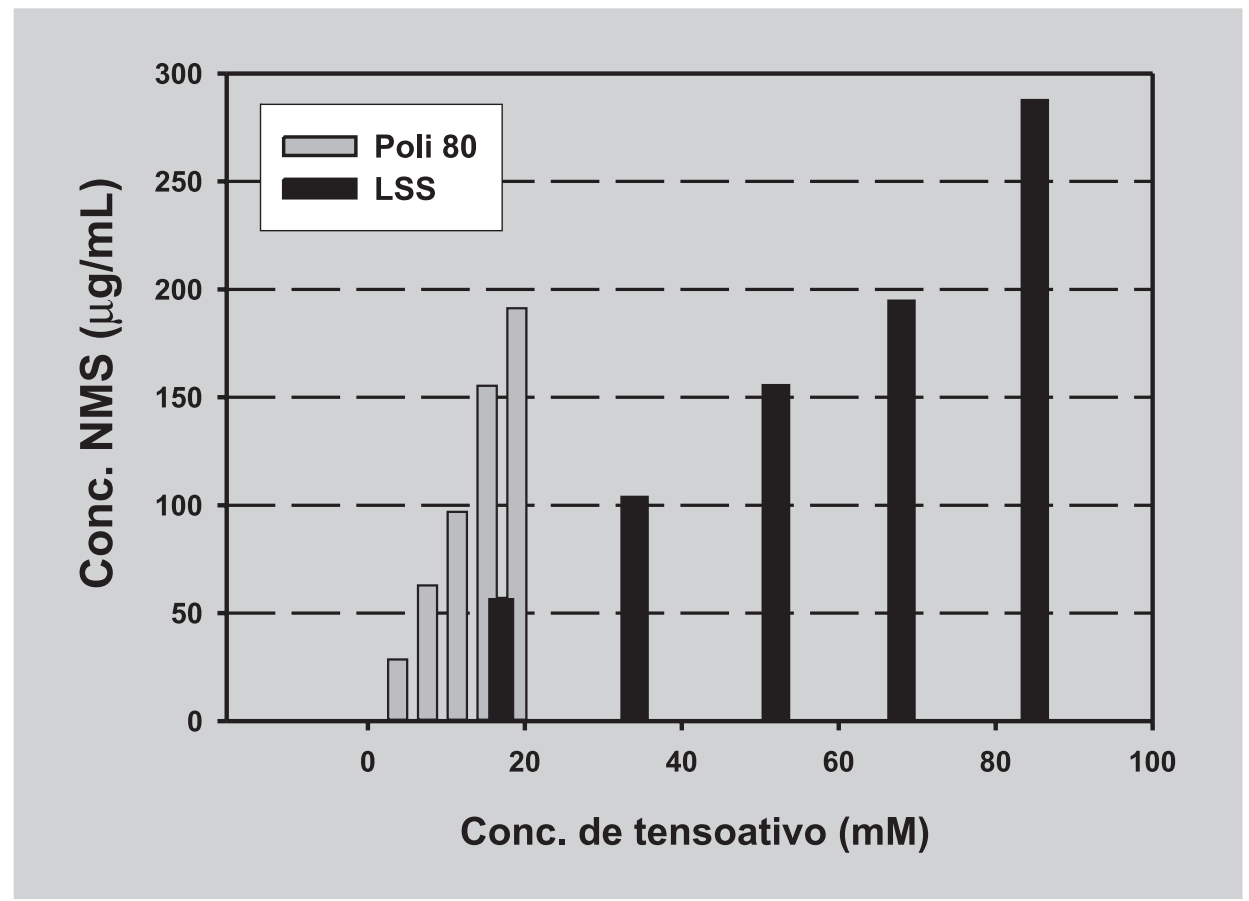

FIGURA 3- Solubilidade da nimesulida (NMS) em soluções aquosas de LSS e Poli $80(0,5 ; 1,0 ; 1,5 ; 2,0$ e 2,5\% p/v para ambos) apresentada em função da concentração molar dos tensoativos.

alcançada em tampão pH 7,4 contendo Poli 80 a 2,5\%.

A concentração de saturação da nimesulida, quer seja nas soluções aquosas de LSS e de Poli 80, aumentou proporcionalmente com o aumento da concentração do tensoativo. Esta concentração está representada em percentagem $(\mathrm{p} / \mathrm{v})$, por ser a maneira mais utilizada pelos compêndios oficiais e literatura da área. Porém, devido ao fato de estes possuirem diferentes pesos moleculares (LSS $=288,38 \mathrm{~g} / \mathrm{mol}$; Poli $80=1310,0 \mathrm{~g} / \mathrm{mol}$ ) (Wade, Weller, 1994), concentrações percentuais iguais não correspondem às mesmas concentrações molares. Com a concentração de tensoativos expressa em $\%(\mathrm{p} / \mathrm{v})$ pode-se, inadequadamente, interpretar que a nimesulida é mais solúvel em soluções aquosas de LSS do que em soluções contendo Poli 80. Porém, analisando-se esta situação em termos de concentração molar, observa-se que, na verdade, soluções aquosas contendo Poli 80 têm maior poder de solubilização da nimesulida, o que pode ser melhor compreendido pela Figura 3.

O lauril sulfato de sódio é um tensoativo aniônico, que, de acordo com a classificação HLB (hydrophiliclipophilic balance), é considerado hidrofílico devido ao seu alto valor de HLB (estimado em 40). O polissorbato 80 , tensoativo não-iônico, é considerado menos hidrofílico que o LSS, por apresentar um valor de HLB igual a 15 (Martin, 1993a; Wade, Weller, 1994). As estruturas de ambos os tensoativos podem ser apreciadas na Figura 4.

Considerando, justamente, a baixa hidrossolubilidade da nimesulida, possuindo, assim, um caráter hidrofóbico e lipofílico ( $\log K=1,3$; coeficiente de partição determinado no sistema clássico $n$-octanol/água), pode-se explicar sua maior afinidade pelas micelas de Poli 80 (menor valor de HLB), com conseqüente maior solubilidade em meios que o continham. O tamanho e o número de micelas também podem influenciar no comportamento geral da pseudofase formada.

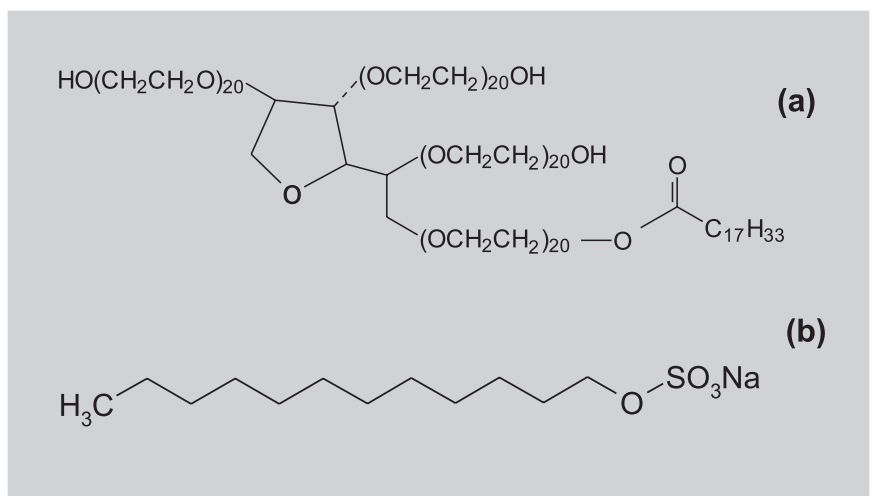

FIGURA 4 - Estrutura química do Poli 80 (a) e do LSS (b). 


\section{Determinação do $\mathrm{pH}$, da tensão superficial e da CMC dos meios}

Os valores de $\mathrm{pH}$ obtidos para os diversos meios variaram de 1,0 a 8,1 e são apresentados na Tabela III.

Os meios de menor e maior solubilização da nimesulida, na ausência ou presença de tensoativo, foram o $\mathrm{HCl} 0,1 N$ e o tampão fosfato $\mathrm{pH} 7,4$, respectivamente. A relação entre a solubilidade da nimesulida e o pH do meio deve-se à crescente ionização da mesma à medida que o $\mathrm{pH}$ aumenta, uma vez que possui caráter fracamente ácido $(\mathrm{pKa}=6,5)$. Através da equação de HendersonHasselbach, calculou-se o percentual de nimesulida na forma básica em $\mathrm{pH} 7,4$, obtendo-se $88,8 \%$ do fármaco na forma desprotonada (ionizada). Como era de se esperar, a elevação do $\mathrm{pH}$ promove maior solubilização da nimesulida. No entanto, é aconselhável utilizar um meio para dissolução com $\mathrm{pH}$ em uma faixa fisiologicamente relevante, ou seja, entre 1,2 e 6,8 . Até mesmo o suco entérico simulado teve seu $\mathrm{pH}$ alterado de 7,5 para 6,8 na última edição da United States Pharmacopeia (2000), face ao questionamento apontado por alguns autores de que aquele valor estaria promovendo superestimação na velocidade de dissolução de ácidos fracos (Galia et al., 1998;
Hörter, Dressman, 2000). Em casos excepcionais, pH superior ainda pode ser justificado, não sendo, entretanto, absolutamente recomendados meios para dissolução que ultrapassem o valor de $\mathrm{pH}$ de 8,0 (CDER/FDA, 1997).

Os valores de tensão superficial obtidos para os meios contendo Poli 80 (36 a $40 \mathrm{mN} / \mathrm{m}$ ) apresentaram-se inseridos na faixa descrita para o suco gástrico; os meios contendo LSS apresentaram tensão superficial (28 a 35 $\mathrm{mN} / \mathrm{m}$ ) abaixo ou próximo ao limite inferior e os meios sem tensoativos forneceram, obviamente, valores superiores. Estes resultados são apresentados na Tabela III.

Os valores de CMC obtidos para as soluções aquosas de LSS, Poli 80, tampão pH 7,4 contendo LSS e tampão $\mathrm{pH} 7,4$ contendo Poli 80 foram, respectivamente, $0,1 \% \mathrm{p} / \mathrm{v} ; 0,05 \% \mathrm{p} / \mathrm{v} ; 0,01 \% \mathrm{p} / \mathrm{v}$ e $0,01 \% \mathrm{p} / \mathrm{v}$. Confirmouse que as concentrações de tensoativo utilizadas estão acima da CMC e, provavelmente, a solubilidade da nimesulida é aumentada pela solubilização da mesma no interior das micelas formadas pelo excesso do tensoativo. A Figura 5 ilustra um gráfico de tensão superficial em função da concentração de tensoativo, indicando a CMC, na inflexão da curva.

Uma vez que a tensão superficial não variou muito, em função de todas as soluções encontrarem-se acima da

TABELA III - Valores de pH e tensão superficial obtidos nos diferentes meios

\begin{tabular}{|c|c|c|}
\hline$\overline{\text { Meios }}$ & pH & Tensão Superficial $(\mathrm{mN} / \mathrm{m})$ média $\pm \mathrm{dp}(\mathrm{n}=3)$ \\
\hline $\mathrm{H}_{2} \mathrm{O}$ ultrapura & 5,12 & $69,8 \pm 0,06^{1}$ \\
\hline LSS $-0,5 \%$ & 8,10 & $35,2 \pm 0,06^{2}$ \\
\hline LSS - $1,5 \%$ & 7,91 & $33,0 \pm 0,06^{3}$ \\
\hline LSS - $2,0 \%$ & 7,82 & $32,2 \pm 0,31^{3}$ \\
\hline Poli $80-0,5 \%$ & 6,88 & $38,8 \pm 0,06^{2}$ \\
\hline Poli $80-1,5 \%$ & 6,45 & $39,4 \pm 0,10^{2}$ \\
\hline Poli $80-2,0 \%$ & 6,43 & $39,3 \pm 0,06^{2}$ \\
\hline $\mathrm{HCl} 0,1 \mathrm{~N}$ & 1,00 & $67,5 \pm 0,32^{1}$ \\
\hline $\mathrm{HCl}$ contendo LSS $-0,5 \%$ & 1,01 & $33,3 \pm 0,10^{3}$ \\
\hline $\mathrm{HCl}$ contendo Poli $80-0,5 \%$ & 0,99 & $36,3 \pm 0,21^{2}$ \\
\hline Tampão pH 5,8 & 5,80 & $69,3 \pm 0,15^{1}$ \\
\hline Tampão pH 5,8 contendo Poli 80 - 1,5 \% & 5,83 & $40,3 \pm 0,26^{2}$ \\
\hline Tampão pH 5,8 contendo Poli 80 - 2,5 \% & 5,83 & $39,5 \pm 0,10^{2}$ \\
\hline Tampão pH 7,4 sem íons $\mathrm{K}^{+}$ & 7,40 & $69,4 \pm 0,31^{1}$ \\
\hline Tampão pH 7,4 contendo LSS - 1,0 \% & 7,47 & $28,5 \pm 0,25^{3}$ \\
\hline Tampão pH 7,4 contendo LSS - 2,0 \% & 7,46 & $28,6 \pm 0,10^{3}$ \\
\hline Tampão pH 7,4 & 7,40 & $66,3 \pm 0,10^{1}$ \\
\hline Tampão pH 7,4 contendo Poli 80 - 1,5 \% & 7,47 & $40,9 \pm 0,10^{2}$ \\
\hline Tampão pH 7,4 contendo Poli 80 - 2,0 \% & 7,46 & $39,8 \pm 0,06^{2}$ \\
\hline Tampão pH 7,4 contendo Poli 80 - 2,5 \% & 7,46 & $38,7 \pm 0,06^{2}$ \\
\hline
\end{tabular}

${ }^{1}$ Valores superiores à faixa fisiológica; ${ }^{2}$ Valores inseridos na faixa fisiológica; ${ }^{3}$ Valores inferiores à faixa fisiológica 


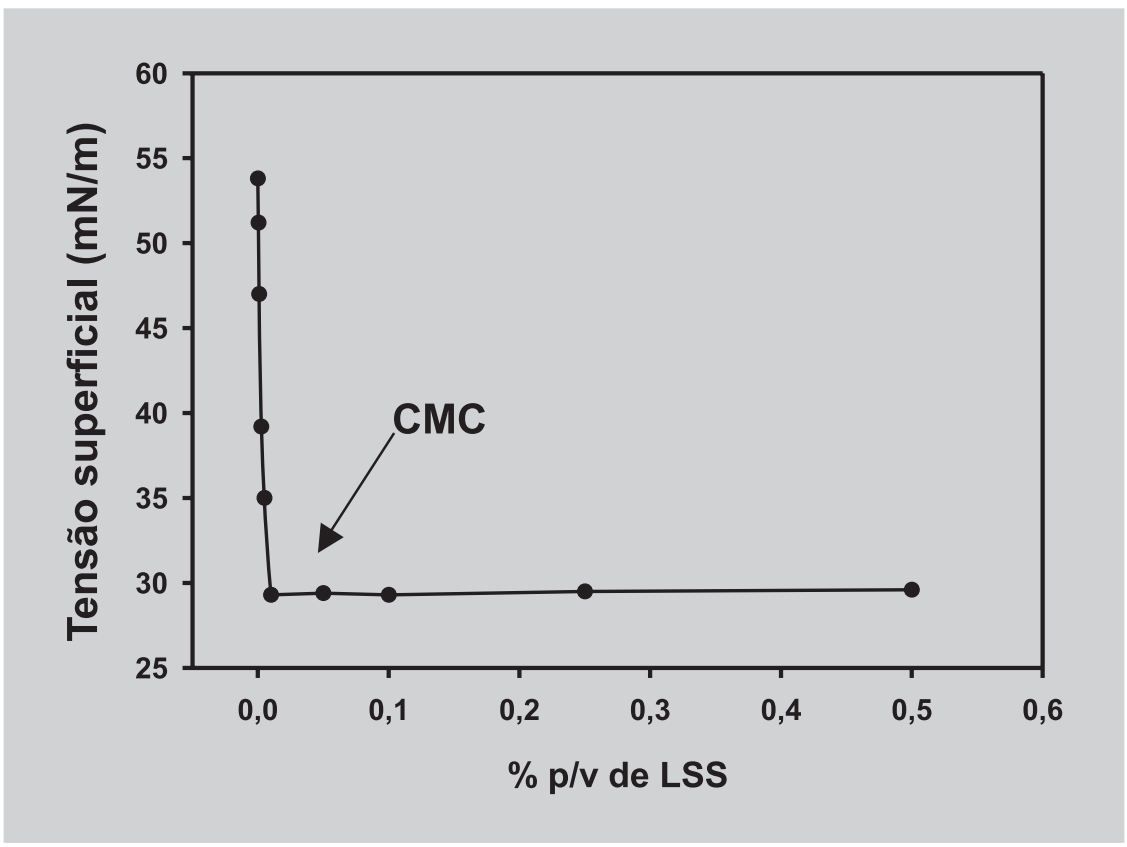

FIGURA 5 - Concentração micelar crítica da solução aquosa de Tampão pH 7,4 contendo LSS.

$\mathrm{CMC}$, o fenômeno da molhabilidade praticamente igualase em todas as condições e teve pequena participação na promoção da solubilidade do fármaco (Schott, Kwan, Feldman, 1982; Schott, 1995).

\section{Avaliação do perfil de dissolução de comprimidos de nimesulida}

Os perfis de dissolução dos três produtos de nimesulida testados, numa avaliação preliminar, são apresentados nas Figuras 6 e 7.
Observa-se que nos meios LSS - 0,5\% (p/v) e Poli $80-1,5 \%(\mathrm{p} / \mathrm{v})$ as três formulações forneceram perfis distintos. Em ambos os meios o Produto B teve sua dissolução superior e o Produto C, inferior; o Produto A apresentou um perfil de dissolução intermediário entre as outras duas formulações. Cabe apontar que o Produto B é o medicamento referência nacional (ANVISA/MS, 2001).

Curiosamente, constata-se que mesmo em meio de baixa solubilidade para o fármaco (Figura 6a), o Produto $\mathrm{B}$ fornece teor de nimesulida dissolvido próximo a $80 \%$ em 15 minutos, do que se depreende que o comprimido
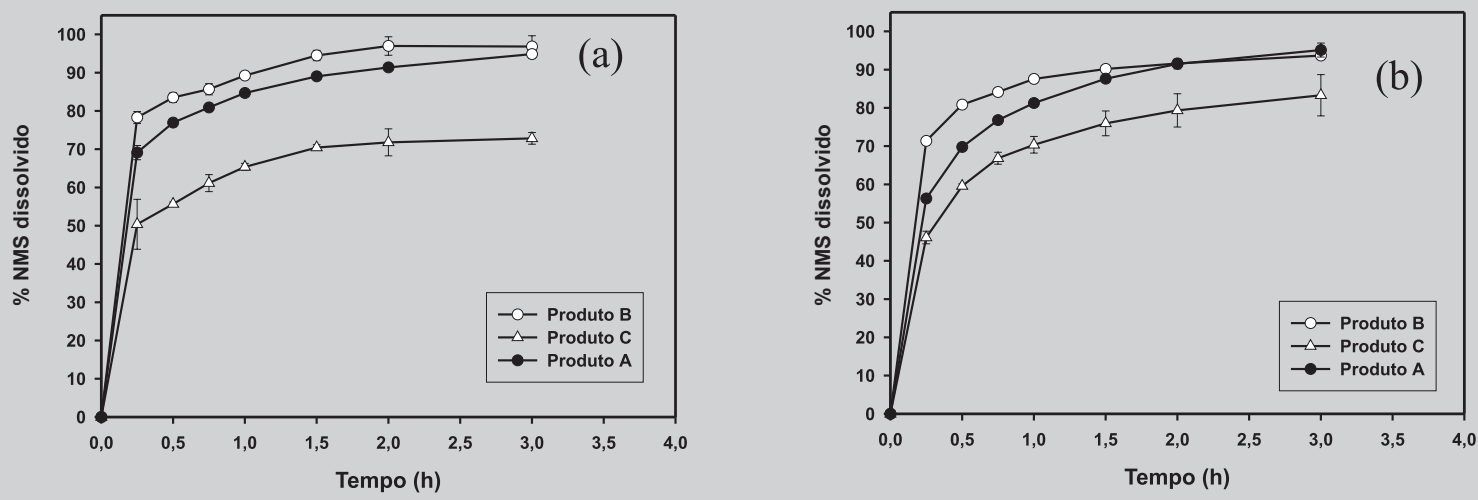

FIGURA 6 - Perfis de dissolução dos Produtos A, B e C: (a) em LSS - 0,5 \% (p/v), a 75 rpm; (b) em Poli 80 - 1,5 \% $(\mathrm{p} / \mathrm{v})$, a $75 \mathrm{rpm}$ (valores médios $\pm \mathrm{dp} ; \mathrm{n}=6$ ). 
$\%$

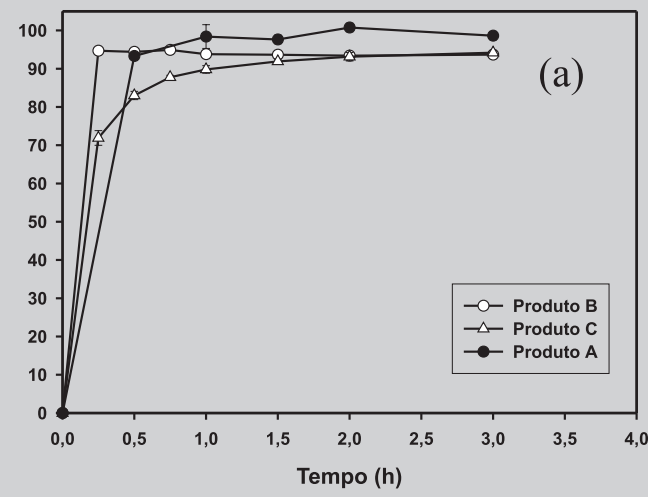

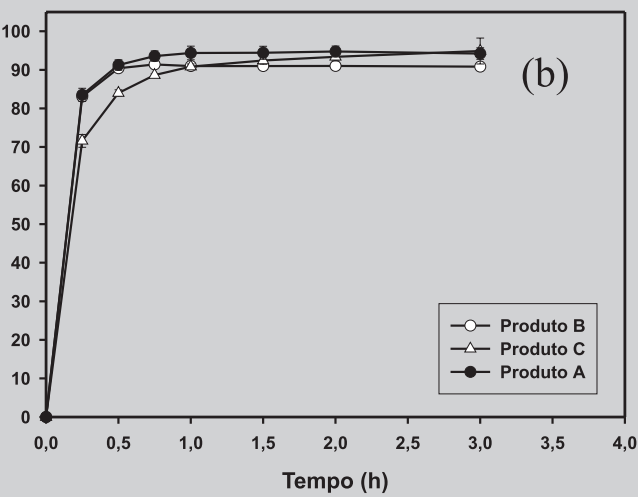

FIGURA 7 - Perfis de dissolução dos Produtos A, B e C em: (a) LSS - 2,0\% (p/v), a 75 rpm; (b) em tampão pH 7,4 contendo Poli $80-2,0 \%(\mathrm{p} / \mathrm{v})$, a $75 \mathrm{rpm}$ (valores médios $\pm \mathrm{dp} ; \mathrm{n}=6$ ).

possui um agente solubilizante (p. ex. excipiente tensoativo) em sua composição, caso contrário, a dissolução do fármaco, nestes níveis, não teria ocorrido. $\mathrm{O}$ pH de 8,1 apresentado por este meio também contribuiu para a rápida dissolução do Produto B. Para o Produto C, este fator por si só não foi suficiente. $\mathrm{O}$ mesmo comportamento não se observa em Poli 80 - 1,5\% (Figura 6b), uma vez que o $\mathrm{pH}$ de 6,5 deste meio, embora mais correspondente ao fisiológico, favorece menos a dissolução de um ácido fraco como a nimesulida.

Os meios avaliados, que promoveram maior solubilização da nimesulida, forneceram perfis de dissolução praticamente sobreponíveis (Figura 7), havendo dissolução de cerca de $90 \%$ do fármaco em 1 hora, para os três Produtos, embora o Produto $\mathrm{C}$ apresente dissolução mais lenta no início do processo. A maior concentração de tensoativo associada ao $\mathrm{pH}(7,4$ e 7,8$)$ evidenciou pouca diferença entre as formulações.

Estes resultados mostram que meios com menor concentração de tensoativo e $\mathrm{pH}<7$ possuem maior habilidade em discriminar diferentes formulações de comprimidos de nimesulida.

\section{CONCLUSÃO}

A solubilidade da nimesulida em soluções aquosas de LSS e Poli 80, em diferentes concentrações \% (p/v), foi superior na presença do primeiro tensoativo. Porém, analisando-se a situação em termos de concentração molar, observa-se que, na verdade, o Poli 80 tem maior poder de solubilização do fármaco, quando comparado com o LSS para concentrações molares equivalentes.
Os tensoativos desempenharam papel fundamental na solubilidade da nimesulida devido à sua baixa hidrossolubilidade, sendo imprescindível a presença dos mesmos nos meios para dissolução. O principal mecanismo de atuação dos tensoativos na promoção da solubilidade da nimesulida é a solubilização micelar.

Os meios aquosos contendo os tensoativos em maior concentração forneceram perfis de dissolução da nimesulida mais rápidos e completos e praticamente sobreponíveis. Porém, a composição destes meios, com altas concentrações de tensoativos, pode estar superestimando a liberação e dissolução do fármaco in vivo. Condições biorrelevantes, isto é, meios de dissolução que apresentam composição e características mais similares aos fluidos do ambiente gastrintestinal deveriam ser mais adotados nos estudos in vitro. Para ácidos fracos, até mesmo um pH entre 7,0 e 7,5 tem sido apontado como fator de superestimação na velocidade de dissolução in vivo.

Somente após um estudo de correlação in vivo-in vitro, as condições para a dissolução de comprimidos de nimesulida poderão ser melhor definidas, necessitando, para isto, a realização de estudos de biodisponibilidade com as formulações envolvidas nestes ensaios preliminares de dissolução.

\section{APOIO FINANCEIRO}

Este trabalho é parte da dissertação de Mestrado de R.L.S. apresentada ao Programa de Pós-Graduação em Ciências Farmacêuticas da Faculdade de Farmácia da UFRJ, com bolsa CAPES. 


\section{ABSTRACT}

\section{Media for nimesulide tablet dissolution: surfactant action}

Nimesulide is a non-steroidal anti-inflammatory drug, which presents low water-solubility and weak-acid properties. The intrinsic solubility of a drug plays an important role on drug dissolution from a solid dosage form. The development of a dissolution test for poorly hydrophilic drugs can exhibit some difficulties and surfactants are often used to promote drug solubilization. In this work the influences of both sodium laurylsulfate and polysorbate 80 on promotion of nimesulide aqueous solubility were studied. The media were also evaluated regarding their $\mathrm{pH}$, surface tension and critical micelle concentration. All media without surfactant gave very low nimesulide solubility $(<60 \mu \mathrm{g} / \mathrm{mL})$ at pH range of 1.0 to 7.4. The higher solubilities $(>300 \mu \mathrm{g} / \mathrm{mL}$ ) were got in phosphate buffer $\mathrm{pH} 7.4$ with polysorbate 80 as additive. As surfactant concentration increases, higher drug saturation values are obtained by micellar solubilization. Dissolution profiles of three nimesulide tablets formulations were conducted employing media with different surfactant concentration by the rotating paddle-method. The results indicated that surfactants in nimesulide dissolution studies should be criteriously chosen in order to avoid poor discriminating medium characteristics.

UNITERMS: Nimesulide. Dissolution. Surfactant. Solubility. Surface tension.

\section{REFERÊNCIAS BIBLIOGRÁFICAS}

ABDOU, H. M. Dissolution. In: Remington: the science and practice of pharmacy. 19. ed. Easton: Mack Publishing Company, 1995. v. 1.p. 593-604.

AMIDON, G. L., LENNERNAS, H., SHAH, V. P., CRISON, J. R. A theoretical basic for a biopharmaceutic drug classification: the correlation of in vitro drug product dissolution and in vivo bioavailability. Pharm. Res., v. 12, n. 3, p. 413-420, 1995.

ANVISA/MS. Disponível em: http://www.anvisa.gov.br/ hotsite/genericos/lista/registrados2.htm. Acesso em: set., 2001.

BERNAREGGI, A. Clinical pharmacokinetics of nimesulide. Clin. Pharmacokinet, v. 35, n. 4, p. 247-274, Oct. 1998.
CÁRCAMO, E. C. Cinética de disolución de medicamentos. Washington: Secretaria General de la Organización de los Estados Americanos, 1981. 103 p.

CDER/FDA . Guidance for industry. Dissolution testing of immediate release solid oral dosage forms. Disponível em: http://www.fda.gov/cder/guidance/index.htm. Acesso em: Ago.,1997. 14 p.

CRISON, J. R., WEINER, N. D., AMIDON, G. L. Dissolution media for in vitro testing of water-insoluble drugs: effect of surfactant purity and electrolyte on in vitro dissolution of carbamazepine in aqueous solutions of sodium lauryl sulfate. J. Pharm. Sci., v. 86, n. 3, p. 384 388, 1997.

DAVIS, R., BROGDEN, R. N. Nimesulide: an update of its pharmacodynamic and pharmacokinetic properties, and therapeutic efficacy. Drugs, v. 48, n. 3,p. 431-454, 1994.

EL-MASSIK, M. A., DARWISH, I. A., HASSAN, E. E., ELKHORDAGUI, L. K. Development of a dissolution medium for glibenclamide. Int. J. Pharm., v. 140, p. 6976, 1996.

FINHOLT, P., SOLVANG, S. Dissolution kinetics of drugs in human gastric juice - the role of surface tension. $J$. Pharm. Sci., v. 57, n. 8, p. 1322-1326, 1968.

GALIA, E., NICOLAIDES, E., HÖRTER, D., LÖBENBERG, R., REPPAS, C., DRESSMAN, J.B.. Evaluation of various dissolution media for predicting in vivo performance of class I and II drugs. Pharm. Res., v.15, p. 698-705, 1998.

HOLLAND, L. A. CHETWYN, N. P., PERKINS, M. D., LUNTE, S. M. Capillary electrophoresis in pharmaceutical analysis. Pharm. Res., v. 14, n. 4, p. 372387, 1997.

HÖRTER, D.; DRESSMAN, J.B. Influence of physicochemical properties on dissolution of drugs in the gastrointestinal tract. Adv. Drug Delivery Rev., v. 46, p. $75-87,2001$

LÖBENBERG, R., AMIDON, G. L. Modern bioavailability, bioequivalence and biopharmaceutics classification system. New scientific approaches to international regulatory standards. Eur. J. Pharm. Biopharm., v. 50, p. 3-12, 2000. 
MARTIN, A. Interfacial Phenomena. In: - Physical pharmacy. 4. ed. Philadelphia: Lea \& Febiger, 1993a. Cap. 14, p. 362.

MARTIN, A. Colloids. In: - Physical pharmacy. 4. ed. Philadelphia: Lea \& Febiger, 1993b. Cap. 15, p. 393.

PIEL, G., PIROTTE, I., DELNEUVILLE. I., NEVEN, P., LIABRES, G., DELARGE, J., DELATTRE, L. Study of the influence of both cyclodextrins and L-lysine on the aqueous solubility of nimesulide: isolation and characterization of nimesulide-L-lysine-cyclodextrin complexes. J. Pharm. Sci., v. 86, p. 475-480, 1997.

SCHOTT, H. Colloidal dispersions. In: Remington: the science and practice of pharmacy. 19. ed. Easton: Mack Publishing Company, 1995. v. 1. p. 252-277.

SCHOTT, H., KWAN, L. C., FELDMAN, S. The role of surfactants in the release of very slightly soluble drugs from tablets. J. Pharm. Sci., v. 71, n. 9, p. 1038-1045, 1982.

SHAH, V. P., NOORY, A., NOORY, C., MCCULLOUGH, B., CLARKE, S., EVERETT, R., NAVIASKY, H., SRINIVASAN, B. N., FORTMAN, D., SKELLY, J. P. In vitro dissolution of sparingly water soluble drug dosage forms. Int. J. Pharm., v. 125, p. 99-106, 1995.
SINGH, S., SHARDA, N., MAHAJAN, L. Spectrophotometric determination of $\mathrm{pK}_{\mathrm{a}}$ of nimesulide. Int. J. Pharm., v. 176, n. 2, p. 261-264, Jan. 1999.

STOREY, D. E. The role of dissolution testing in the design of immediate release dosage forms. Drug Inf. J., v. 30, p. 1039-1044, 1996.

UNITED STATES PHARMACOPEIA. 24 ed. Rockville: U.S.P. Convention, 2000. 2569 p.

WADE, A., WELLER, P. J. ed. Handbook of pharmaceutical excipients. 2. ed. London: The Pharmaceutical Press, 1994. p. 375-378, $448-450$.

ZOGRAFI, G. Interfacial phenomena. In: Remington: the science and practice of pharmacy. 19. ed. Easton: Mack Publishing Company, 1995. v. 1. p. 241-251.

Recebido para publicação em 10 de outubro de 2001. 